Please do not remove this page

RMIT

UNIVERSITY

\title{
Nanoscale characterization of energy generation from piezoelectric thin films
}

Bhaskaran, Madhu; Sriram, Sharath; Ruffell, Simon; Mitchell, Arnan

https://researchrepository.rmit.edu.au/esploro/outputs/9921857836001341/filesAndLinks?institution=61RMIT_INST\&index=null

Bhaskaran, M., Sriram, S., Ruffell, S., \& Mitchell, A. (2011). Nanoscale characterization of energy generation from piezoelectric thin films. Advanced Functional Materials, 21(12), 2251-2257.

https://doi.org/10.1002/adfm.201002663

Document Version: Accepted Manuscript

Published Version: https://doi.org/10.1002/adfm.201002663

Repository homepage: https://researchrepository.rmit.edu.au

(c) 2011 WILEY-VCH Verlag GmbH \& Co. KGaA, Weinheim

Downloaded On 2023/04/26 22:49:09 +1000

Please do not remove this page 
Thank you for downloading this document from the RMIT Research Repository.

The RMIT Research Repository is an open access database showcasing the research outputs of RMIT University researchers.

RMIT Research Repository: http://researchbank.rmit.edu.au/

\section{Citation:}

Bhaskaran, M, Sriram, S, Ruffell, S and Mitchell, A 2011, 'Nanoscale characterization of energy generation from piezoelectric thin films', Advanced Functional Materials, vol. 21, no. 12, pp. 2251-2257.

See this record in the RMIT Research Repository at:

http://researchbank.rmit.edu.au/view/rmit:11524

Version: Accepted Manuscript

Copyright Statement: (C) 2011 WILEY-VCH Verlag GmbH \& Co. KGaA, Weinheim

Link to Published Version:

http://dx.doi.org/10.1002/adfm.201002663 
/DOI: 10.1002/adfm.201002663

Submitted to

\section{Nanoscale Characterization of Energy Generation from Piezoelectric Thin Films}

By Madhu Bhaskaran, ${ }^{*} \S$ Sharath Sriram, ${ }^{\S}$ Simon Ruffell, and Arnan Mitchell

[*] Dr. Madhu Bhaskaran, Dr. Sharath Sriram, Prof. Arnan Mitchell

Microplatforms Research Group and Platform Technologies Research Institute, School of Electrical and Computer Engineering

RMIT University, GPO Box 2476, Melbourne, Victoria 3001 (Australia)

E-mail:madhu.bhaskaran@gmail.com,madhu.bhaskaran@rmit.edu.au

Dr. Simon Ruffell

Department of Electronic Materials Engineering, Research School of Physics and Engineering Australian National University, Australian Capital Territory 0200 (Australia)

[§] These authors contributed equally to this work.

Keywords: energy generation, piezoelectric thin films, nanoislands, in situ electromechanical characterization, size effects

We report on the use of nanoindentation to characterize in situ the voltage and current generation of piezoelectric thin films. This work presents the controlled observation of nanoscale piezoelectric voltage and current generation, allowing accurate quantification and mapping of force function variations. We characterize both continuous thin films and lithographically patterned nanoislands with constrained interaction area. The influence of size on energy generation parameters is reported demonstrating that nanoislands can exhibit more effective current generation than continuous films. These quantitative finding suggests that further research into the impact of nanoscale patterning of piezoelectric thin films may yield an improved materials platform for integrated microscale energy scavenging systems.

\section{Introduction}

Highly efficient piezoelectric thin films, which can convert small mechanical movements into voltages on the scale of silicon transistor logic, could enable micro- and nano-electronic circuits which can scavenge their power from their environment. ${ }^{[1-3]}$ Such devices have potential applications for low power portable and implantable biomedical sensors and devices. 
Submitted to 14 A

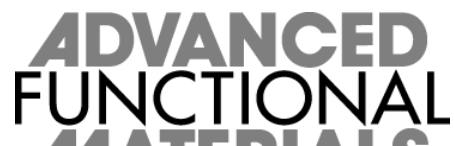

The use of $\mathrm{ZnO}$ and $\mathrm{BaTiO}_{3}$ nanowires has seen a significant advance in micro-scale piezoelectric energy scavenging, relying on a combination of semiconductor and piezoelectric properties. ${ }^{[2-6]}$ The ability to characterize the nanoscale electromechanical properties of these nanowires was instrumental in developing innovative designs to demonstrate energy harvesting capabilities ${ }^{[4,5]}$ Energy generation from piezoelectric thin films on flexible substrates has also been demonstrated, ${ }^{[7]}$ with greater potential for integration with mature electronic and micromechanical manufacturing. To realize this practical thin film energy scavenging technology, techniques for electro-mechanical characterization of thin films is critical and such procedures must be conducted on the nano-scale, both in terms of the film thickness and the lateral geometries into which these films may be lithographically patterned.

In this paper we report on the use of nanoindentation to characterize in situ the voltage and current generation of piezoelectric thin films. This novel nanoindentation approach enables the controlled observation of nanoscale piezoelectric voltage and current generation. We utilize this technique to quantify and study the influence of force functions on piezoelectric energy generation. Size effects are also investigated by comparing thin films and lithographically patterned nanoislands, identifying the dependence of piezoelectric energy generation on thickness and area.

\section{Piezoelectric Thin Films and Nanoislands}

Piezoelectric thin film samples were synthesized by RF magnetron sputter deposition of PSZT of composition $\left(\mathrm{Pb}_{0.92} \mathrm{Sr}_{0.08}\right)\left(\mathrm{Zr}_{0.65} \mathrm{Ti}_{0.35}\right) \mathrm{O}_{3}$ onto metalized silicon substrates. This composition has been demonstrated to produce the highest piezoelectric response for a thin film on a silicon substrate reported to date. ${ }^{[8,9]}$ A $200 \mathrm{~nm}$ platinum coating with a $20 \mathrm{~nm}$ titanium dioxide adhesion layer was used to form the conductive bottom electrode metallization of the silicon substrates. PSZT thin films were sputtered for duration of 
Submitted to 14 A

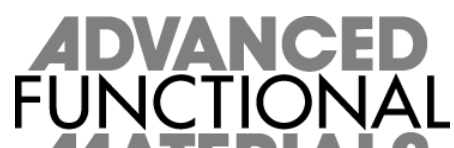

2 and 4 hours resulting in thin films of thicknesses $700 \mathrm{~nm}$ and $1400 \mathrm{~nm}$, respectively (see Supporting Information).

Cross-sectional transmission electron microscopy analysis of PSZT thin film samples revealed the existence of a highly ordered columnar grain structure, as shown in Figure 1(a). Hollow cone illumination revealed that a large number of grains share similar orientation. The temperature of PSZT deposition results in grain growth in the platinum bottom electrode layer (deposited at room temperature), resulting in preferential (111) orientation of platinum. X-ray diffraction analysis [Figure 1(b)] confirmed that the films were preferentially oriented and the growth direction was aligned strongly to the $c$-axis of the crystal structure. ${ }^{[10]}$

It was anticipated that a comparison of the properties of continuous thin films and isolated nanoislands would offer insights into the nanoscale piezoelectric behavior of the film. Piezoelectric nanoislands were defined by a combination of electron beam lithography and lift-off patterning (see Supporting Information). Electron beam resist coating, on silicon substrates metalized as above, was patterned using a field emission gun scanning electron microscope (FEI Nova NanoSEM) to define circular openings varying in diameter from 100-500 nm. Deposition of $\sim 200 \mathrm{~nm}$ thick films PSZT was carried out at room temperature followed by lift-off with chlorobenzene. This resulted in well defined piezoelectric nanoislands, which were either hemispherical or cylindrical depending on the aspect ratio of the island. PSZT thin films deposited at room temperature are generally nanocrystalline. In order to initialize grain growth and attain preferential orientation, the samples were then annealed for 60 minutes in a 10\% oxygen partial pressure (argon balance) atmosphere at $650{ }^{\circ} \mathrm{C}$. These conditions were chosen to replicate the high temperature deposition of thin 
Submitted to

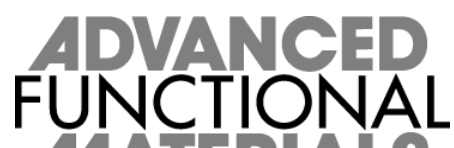

MATERIALS

film samples. ${ }^{[1]]}$ The resulting array of piezoelectric nanoislands is shown in Figure 2, from

which islands with diameter of 200 and $400 \mathrm{~nm}$ were chosen for testing.

\section{In Situ Nanoelectromechanical Characterization}

Nanoindentation with in situ electrical characterization was performed to characterize the nanoscale electromechanical properties of the piezoelectric thin films and nanoislands.

Testing was performed using a Hysitron Triboindenter, which was configured to perform in situ electrical measurements using a conductive Berkovich tip (boron-doped diamond with resistivity of $3.3 \Omega \mathrm{cm}$ ). The tip-contact resistance varies as a function of the penetration depth, as the interaction area increases. ${ }^{[12-13]}$ This configuration termed NanoECR ${ }^{\mathrm{TM}}$ enables measurement of the open circuit voltage and short circuit current generation during nanoindentation (see Supporting Information). ${ }^{[12-13]}$ Nanoindentation was performed under load control with maximum loads ranging from 0.1 to $10 \mathrm{mN}$. Figure 3 shows a schematic of the NanoECR system configured for open circuit voltage measurements during nanoindentation testing. This approach to characterise nanoscale energy generation parameters compares to in situ measurements by Rar and co-workers under the converse piezoelectric effect. $^{[14]}$

Figure 4 shows typical load-unload curve data for indents made in the 700 and $1400 \mathrm{~nm}$ thick PSZT films. The mechanical response is purely elastic for loads of $2.5 \mathrm{mN}$ and below, with contributions from plastic deformation apparent at higher loads. The 'kink' in the loading curve at around $3 \mathrm{mN}$ may be an indication of the plastic deformation. The early loading characteristics for the thicker films differ from those of the $700 \mathrm{~nm}$ film. A substantial tip displacement is observed until a load of $\sim 1 \mathrm{mN}$ is reached. This penetration corresponds roughly to the surface roughness of the thick film [see Figure 1(a)]. The surface roughness is less for the thin film, thus resulting in much more reproducible load/unload indentation curves. 
Submitted to

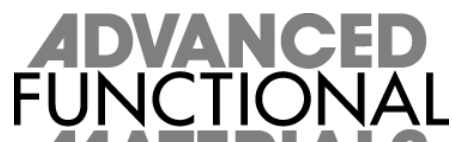

MATERIALS

However, the mechanical response following this initial region is approximately the same for both films. For the figures shown, the maximum tip displacements were $120 \mathrm{~nm}$ and $220 \mathrm{~nm}$, for the $700 \mathrm{~nm}$ and $1400 \mathrm{~nm}$ PSZT films, respectively. These maximum tip displacements correspond to induced strains in the range of $15-17 \%$ for both cases. Interestingly, despite the variability in mechanical response, the generated electrical signatures are very reproducible (as shown later). An example of repeat loading and unloading is also shown in Figure 4(c), where following the initial load-unload cycle where plastic deformation occurs, the PSZT film exhibits an elastic response for the subsequent load-unload cycles.

The voltage measured as a function of applied force for the $700 \mathrm{~nm}$ film is presented in Figure 5, with an impulse force [Figure 5(a)] and for sustained force [Figure 5(b)]. Two immediate trends in the nanoscale voltage generation were observed. Firstly, the voltage produced is approximately linear with applied force and secondly, the voltage is sustained when the applied force was held constant. While piezoelectric voltage generation is expected only for changes in force, the sustained voltage observed in the latter figure can be attributed to charge storage due to the extremely high dielectric constant of PSZT ( $\varepsilon_{\mathrm{r}}$ of $\left.1200-1600\right)$. Gradual decay of the generated voltage as a result of these capacitive properties can be observed on closer examination.

\section{Piezoelectric Voltage Generation and Size Effects}

Figure 6 compares measured voltage levels for PSZT thin films of the two different thicknesses, and shows that larger voltages are generated for the thicker $1400 \mathrm{~nm}$ film. The width of the nanocolumns in the PSZT increases with thickness. This greater grain size probably results in greater voltage generation in the $1400 \mathrm{~nm}$ thicker films, as the indented region has greater probability of compressing identically oriented grains.

$V \quad=\quad 4.07 F+7.99$ 
Submitted to

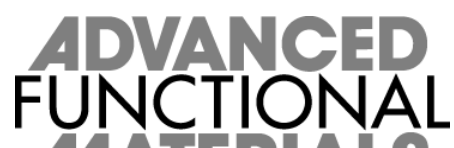

MATERIALS

The linear relationship of the applied force to the generated voltage can be descibed by equation (1), where $F$ is the applied force in $\mathrm{mN}$ and $V$ is the generated voltage in $\mathrm{mV}$. The equation is extracted based on the data in Figure 6 for the $700 \mathrm{~nm}$ thick film. The slope for the linear expression in (1) is $22 \%$ larger for the $1400 \mathrm{~nm}$ film.

The influence of different force function on voltage generation for these thicker films is shown in Figure 7. These results clearly demonstrate the repeatability of voltage generation. Consistent values of generated voltages were measured after multiple force cycles, with minimal hysteresis observed in the results. In the case of multiple loading/unloading cycles, the PSZT films respond elastically to the indentation following the first cycle [an example of this behavior is shown in Figure 4(c)]. However, the generated voltage at the maximum load is the same for all indentation cycles. This behavior suggests that the plastic deformation induced by the indentation during the first cycle does not result in voltage generation, which in turn suggests that voltage generation is due only to elastic deformation. The plastic deformation possibly originates from slip dislocations from the highly nanocolumnar structures, while the elastic deformations relates to compression of the lattice and the resulting piezoelectric potential. Further work is required to investigate the nature of the plastic deformations and related nanomechanical properties of these films. It should be noted that such deformations have been reported to influence local polarization in ferroelectrics, ${ }^{[15-18]}$ but that the PSZT composition under study is a relaxor ferroelectric.

Mapping of consistency in voltage generation across the thin film sample was also performed [Figure $7(\mathrm{~d})]$. Line maps for three different forces $(0.1,2.5$, and $5.0 \mathrm{mN})$ are shown in [Figure 7(d)]. Such mapping enables identification of variations in relation to thin film microstructure (originating from preferential but polycrystalline structure of the films). The 
Submitted to

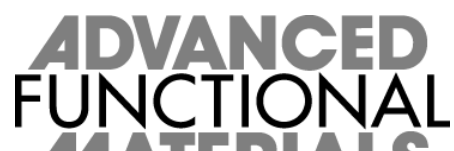

MATERIALS

maximum variation from average voltages measured for 2.5 and $5.0 \mathrm{mN}$ forces were $2.4 \%$ and $5.4 \%$, respectively.

To investigate the impact of lateral confinement of the nanocrystals, the $700 \mathrm{~nm}$ film was patterned to form 200 and $400 \mathrm{~nm}$ islands. Tests were carried out with low forces of 0.1 and $2.5 \mathrm{mN}$. Loading at and above this $2.5 \mathrm{mN}$ threshold induced severe plastic deformation in some cases for the $200 \mathrm{~nm}$ diameter islands. Measured values for voltage generation for both the $200 \mathrm{~nm}$ and $400 \mathrm{~nm}$ islands were very similar ( 4 and $\sim 22 \mathrm{mV}$ for 0.1 and $2.5 \mathrm{mN}$ of force) and matched those for the $700 \mathrm{~nm}$ PSZT thin films. These results are summarized in Table 1. This demonstrated that the planar geometry had no influence on the voltage generation.

\section{Piezoelectric Current Transients and Size Effects}

Having performed rigorous characterization of nanoscale voltage generation, preliminary current generation experiments were carried out in the short circuit mode. Unlike the voltage which was proportional to the applied of force, current was expected to be related to the changes in the applied force. Figure 8 presents the current measured during indentation. Peaks in current are observed at abrupt changes in the applied force as expected, with three peaks observed in Figure 8(a) at the three abrupt changes in force (the application of force, switching of force from compression to relaxation, and return to zero force). The polarity of current also varies, often with negative current produced during film compression (application of force) and positive current produced during removal of force. This was along expected lines, unlike voltage which was influenced by capacitive effects, with the origin of these transient currents and their polarities clearly identified by earlier work by Wang et al. ${ }^{[6]}$ Current transient trends with large forces up to $500 \mathrm{mN}$ have also been reported, with their 
Submitted to

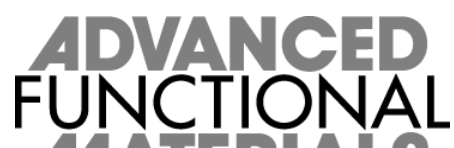

influence on ferroelectric depolarization investigated. ${ }^{[15,16]}$ The current transients for even the largest forces in these reports were almost two order of magnitude smaller.

Figure 8(a) shows an example of one such set of current transients. The value of current generated varies from $40 \mathrm{pA}$ up to a maximum of $\sim 170 \mathrm{pA}$ depending on the sample under study and the force function. The repeatability of transient current generation was verified for multiple load cycles [Figure 8(b)], corresponding to voltage generation shown in Fig. 7(b), with repeatable transients with magnitude of $\sim 100 \mathrm{pA}$ generated. Based on the measurements of current transients, which are related to charge generation, the average piezoelectric charge constant $d_{33}$ was determined ${ }^{[19]}$ to be $\sim 810 \mathrm{pC} \mathrm{N}^{-1}$. This value, albeit large, corresponds very well to the maximum $d_{33}$ of $892 \mathrm{pm} \mathrm{V}^{-1}$ for PSZT thin films measured under the inverse piezoelectric effect. ${ }^{[9]}$

This value of the current transients observed was dependent on both the magnitude of the force and was significantly influenced by the planar geometry of the piezoelectric being stressed (unlike voltage generation). The relationship between the sample and current generated is shown in Table 2 (as an average of two measurements). The larger current densities in restricted geometries such as the nanoislands are probably due to the lack of surrounding material, which is a potential leakage path for generated current.

In addition to the limited leakage paths in the case of the nanoislands, size dependent flexoelectric effects could play a role in increased piezoelectric energy scavenging. Majdoub et al. have analytically demonstrated that piezoelectric energy harvesting is enhanced at the nanoscale due to increased strain gradients, which scales with the feature size of nanostructures. ${ }^{[20-22]}$ We have also shown by a combination of experimental measurements 
Submitted to

and phase field simulations that surface nanostructure leads to piezoelectric strain and response enhancment. ${ }^{[23]}$ These results highlight the need for further study into the relationship between strain gradients in nanostructures and piezoelectric response.

\section{Influence of Nanoindentation Loading Rate}

As expected and also evident in Figure 8, piezoelectric current generation is related to changes in applied force. Thus to further assess the energy generation properties of the nanostructured piezoelectric, the effect of varying the force loading rate was investigated.

Table 3 provides a snapshot of the results of this investigation (with details provided in the Supporting Information). The impact of loading rate on peak voltage generation is minimal as expected. A slight increase is noted for more rapid loading which may be due to transient effects. Conversely, the peak current increases almost in proportion to the rate of loading. The magnitude of the current transient $I$ generated (in $\mathrm{pA}$ ) as a function of the rate of change applied force $F$ (in $\mathrm{mN} \mathrm{s}^{-1}$ ) is depicted mathematically by equation (2). Considering these results and the characteristics of Figure 8, it is suggested that the approach demonstrated in this paper should be used to further study the influence of this parameter, including establishing the upper frequency limit of such direct force piezoelectric energy generation.

$$
|I|=1.83 \frac{d^{2} F}{d t^{2}}-3.50 \frac{d F}{d t}+21.67
$$

\section{Conclusions}

We have demonstrated an approach for in situ nanoelectromechanical characterization of piezoelectric thin films. These results represent one of the first demonstrations of in situ nanoscale electromechanical characterization of piezoelectric thin films. The technique presented in this work can be readily extended to combine charge measurements as a means of determining the nanoscale piezoelectric response under the direct effect. 
Submitted to

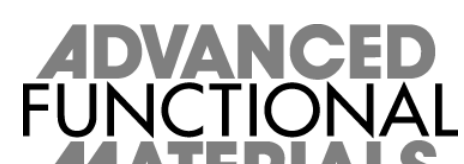

The results from this investigation reinforce the potential for use of piezoelectric thin films for low frequency energy scavenging. Moreover, results from area dependence of voltage and transient current generation show promise for use of micro-fabricated piezoelectric thin films structures in energy harvesting devices. The voltage output of up to $\sim 40 \mathrm{mV}$ and current transients up to $\sim 200 \mathrm{pA}$, compare very well to published results for nanowire generators of $\sim 30 \mathrm{mV}$ and $\sim 100 \mathrm{pA}$ which were shown to be suitable for powering nanosensors. ${ }^{[24]}$ The effective power generated is $\sim 250 \mu \mathrm{W} \mathrm{mm} \mathrm{m}^{-2}$ at $5.0 \mathrm{mN}$ force.

The independence of voltage generation and enhanced current generation observed on constrained planar geometries indicates potential for capitalizing on current nanofabrication advances, to potentially increase current density. The capability to analyze current and voltage generation from nano-scale structures will enable further research into nano-structured thin film arrays required to achieve piezoelectric energy harvesting micro-devices.

\section{Acknowledgements}

The authors acknowledge funding support from the Australian Research Council (Discovery Project DP1092717), the Australian Institute of Nuclear Science and Engineering, the CASS Foundation, and RMIT University (Emerging Researcher Grant). The authors also thank Dr. Gorgi Kostovski for assistance with the electron beam lithography. Supporting Information is available online from Wiley InterScience or from the author.

Received: ((will be filled in by the editorial staff))

Revised: ((will be filled in by the editorial staff)) Published online: ((will be filled in by the editorial staff))

_[1] S. Roundy, P. K. Wright, J. M. Rabaey, Energy Scavenging for Wireless Sensor

Networks, Kluwer Academic, Boston 2003.

[2] Z. L. Wang, Adv. Funct. Mater. 2008, 18, 3553.

[3] R. Yang, Y. Qin, C. Li, Guangzhu, Z. L. Wang, Nano Lett. 2009, 9, 1201.

[4] Z. L. Wang, J. Song, Science 2006, 312, 242. 
Submitted to

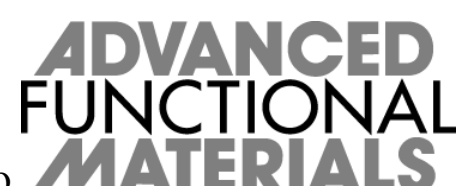

[5] J. H. Song, J. Zhou, Z. L. Wang, Nano Lett. 2006, 6, 1656.

_[6] X. Wang, J. Song, J. Liu, Z. L. Wang, Science 2007, 316, 102.

_[7] Z. Wang, J. Hu, A. P. Suryavanshi, K. Yum, M.-F. Yu, Nano Lett. 2007, 7, 2966.

[8] S. Sriram, M. Bhaskaran, A. S. Holland, K. T. Short, B. A. Latella, J. Appl. Phys. 2007, $101,104910$.

[9] S. Sriram, M. Bhaskaran, A. Mitchell, Scripta Mater. 2010, 63, 189.

[10] M. Bhaskaran, S. Sriram, D. R. G. Mitchell, K. T. Short, A. S. Holland, Thin Solid Films 2008, 516, 8101.

_[11] S. Sriram, M. Bhaskaran, J. du Plessis, K. T. Short, V. P. Sivan, A. S. Holland, Micron 2009, 40, 104.

[12] S. Ruffell, J. E. Bradby, J. S. Williams, O. L. Warren, J. Mater. Res. 2007, 22, 578.

_[13] S. Ruffell, J. E. Bradby, N. Fujisawa, J. S. Williams, J. Appl. Phys. 2007, 101, 083531.

[14] A. Rar, G. M. Pharr, W. C. Oliver, E. Karapetian, S. V. Kalinin, J. Mater. Res. 2006, 21,552 .

[15] M. Algueró, A. J. Bushby, M. J. Reece, R. Poyato, J. Ricote, M. L. Calzada, L. Pardo, Appl. Phys. Lett. 2001, 79, 3830.

[16] V. Koval, M. J. Reece, A. J. Bushby, J. Appl. Phys. 2005, 97, 074301.

[17] G. A. Schneider, T. Scholz, J. Muñoz-Saldaña, M. V. Swain, Appl. Phys. Lett. 2005, $86,192903$.

_[18] G. A. Schneider, T. Scholz, F. J. Espinoza-Beltrán, Appl. Phys. Lett. 2008, 92, 022906.

[119] S. Sridhar, A. E. Giannakopoulos, S. Suresh, J. Appl. Phys. 2000, 87, 8451.

[20] M. S. Majdoub, P. Sharma, T. Cagin, Phys. Rev. B 2008, 77, 125424.

[21] M. S. Majdoub, P. Sharma, T. Cagin, Phys. Rev. B 2008, 78, 121407(R).

[22] J. Cao, J. Wu, Mater. Sci. Eng. R 2011, 71, 35. 


\section{Submitted to \\ FUNCTIONAL}

[23] S. Sriram, M. Bhaskaran, R. Ahluwalia, T. G. Nguyen, N. Ng, D. J. Srolovitz, K.

Kalantar-zadeh, A. Mitchell, ACS Nano 2011, Article ASAP (doi: 10.1021/nn103561u).

[24] R. Yang, Y. Qin, C. Li, G. Zhu, Z. L. Wang, Nano Lett. 2009, 9, 1201. 


\section{Submitted to}

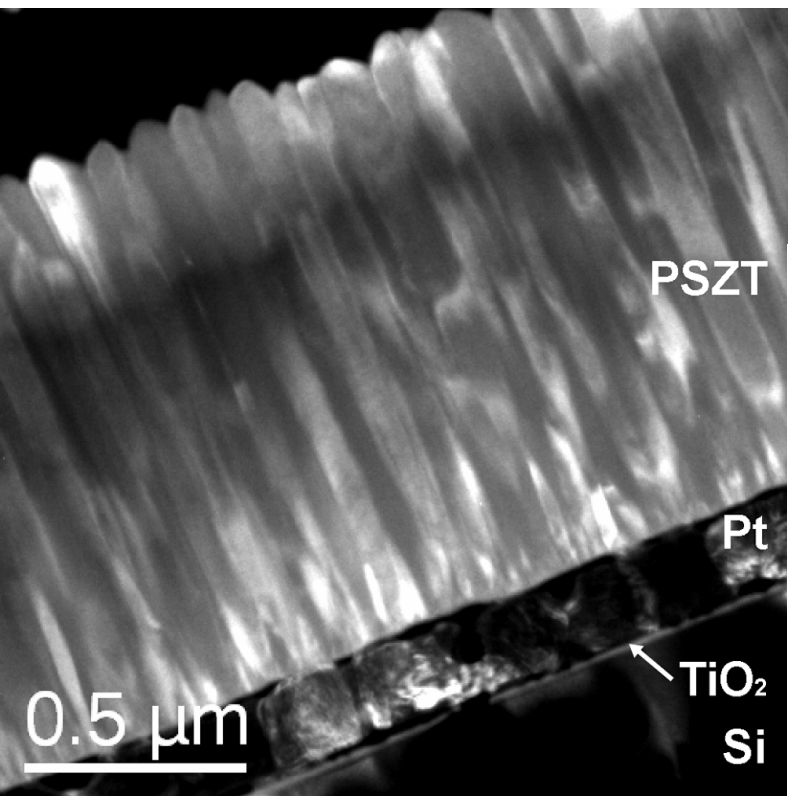

(a)

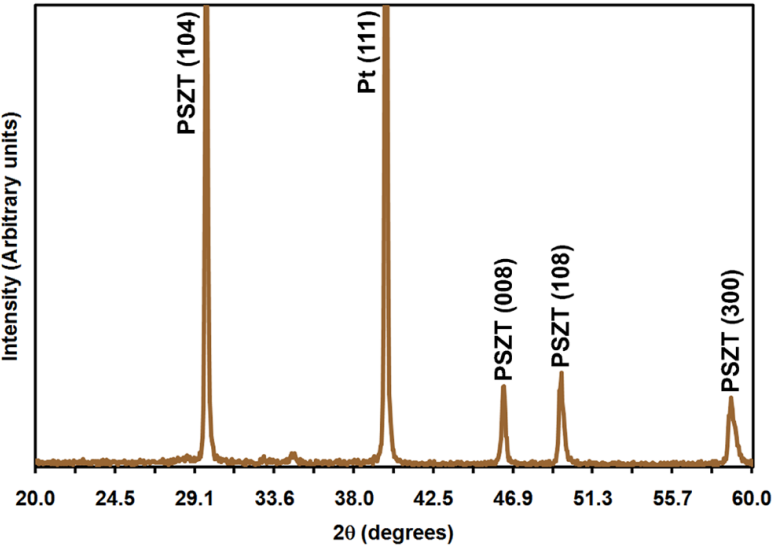

(b)

Figure 1. Microstructural characterization of PSZT thin films deposited on $\mathrm{Pt} / \mathrm{TiO}_{2} / \mathrm{Si}$ substrates: (a) Hollow cone dark field cross-sectional transmission electron micrograph showing columnar grain growth and (b) X-ray diffractogram indicating preferential (104) orientation.

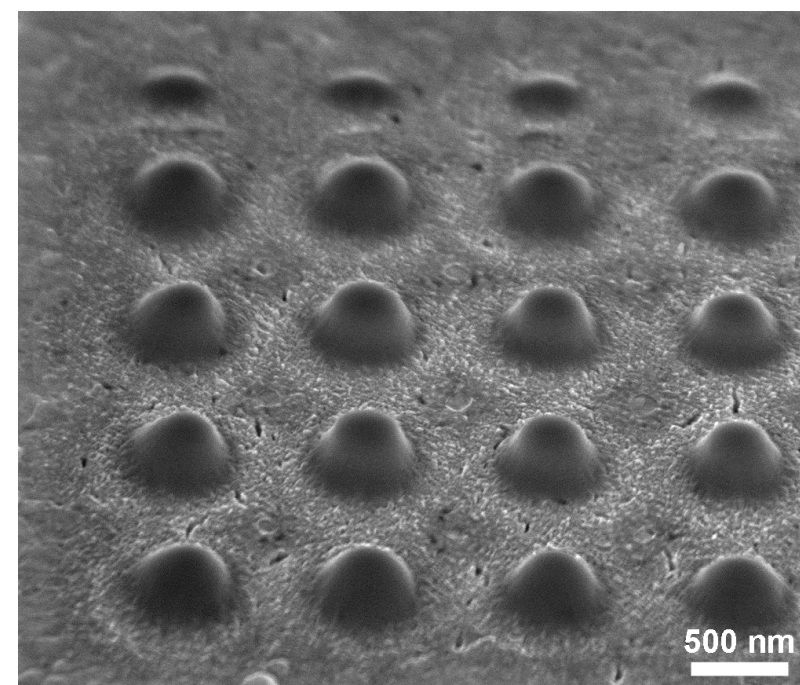

(a)

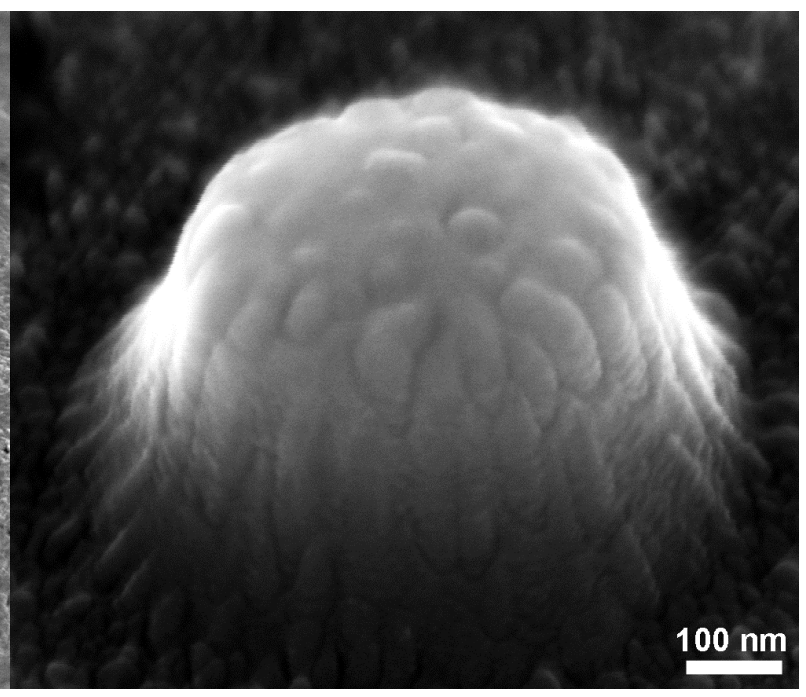

(b)

Figure 2. Scanning electron micrographs of arrays of PSZT nanoislands (imaged at $45^{\circ}$ ): (a) 400 nm diameter nanoislands and (b) detail of a nanoisland showing grain structure. The substrate roughness observed is due to the crystallization of the platinum electrode layer into the (111) low energy state during annealing. 


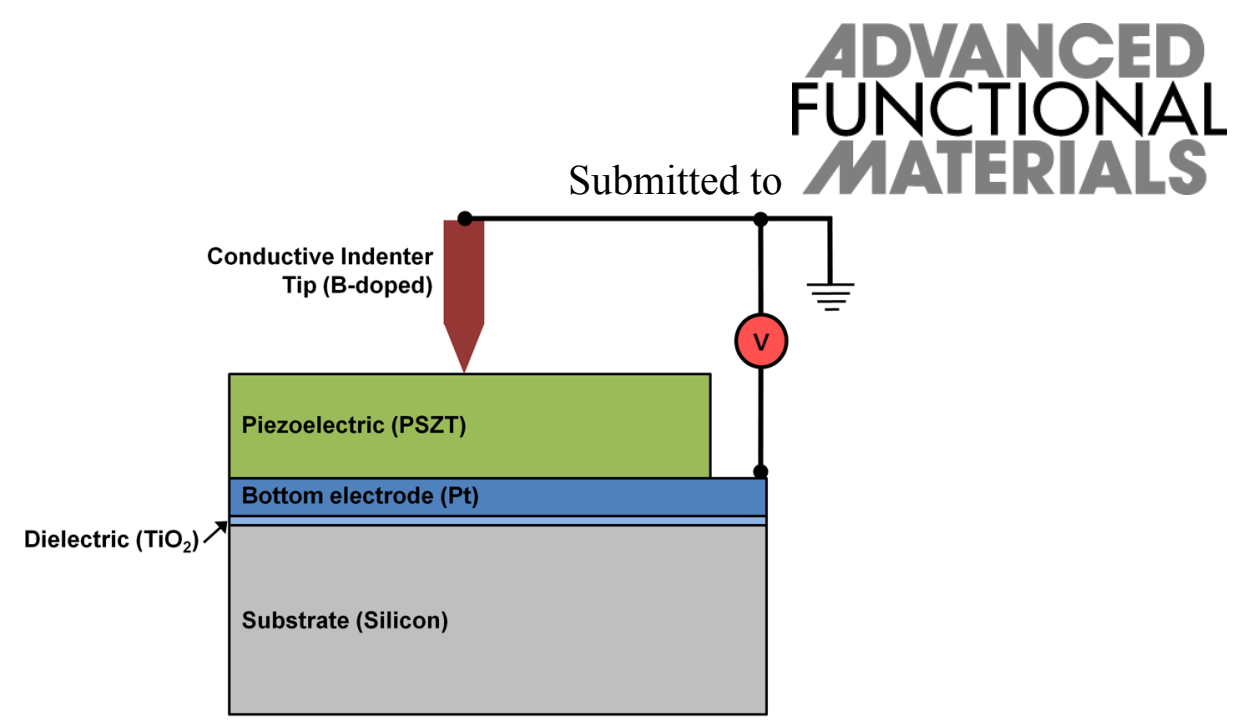

Figure 3. Schematic representation of setup used for electrical characterization during nanoindentation. The open circuit configuration used for measuring voltage between the electrically grounded indenter tip and the bottom electrode of platinum is shown. (Not to scale.)

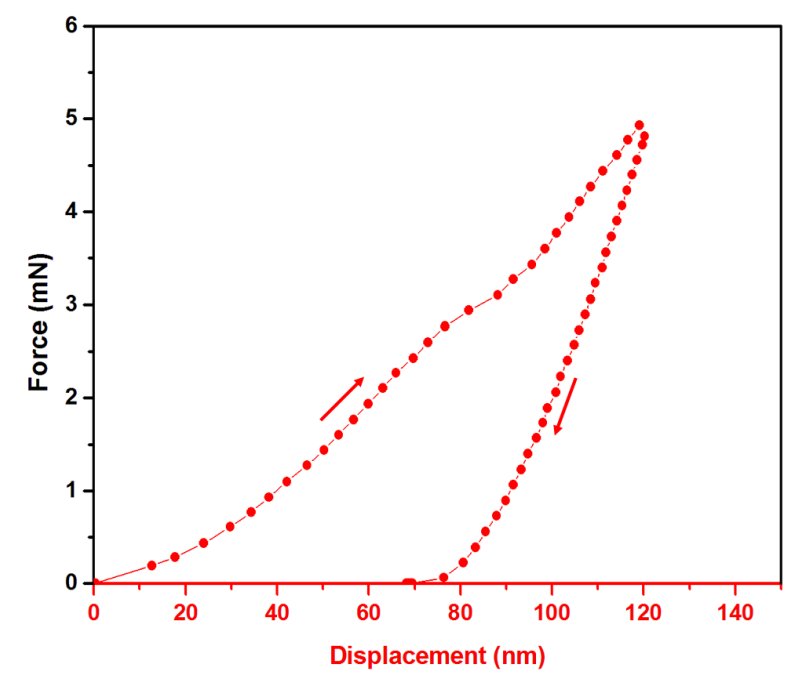

(a)

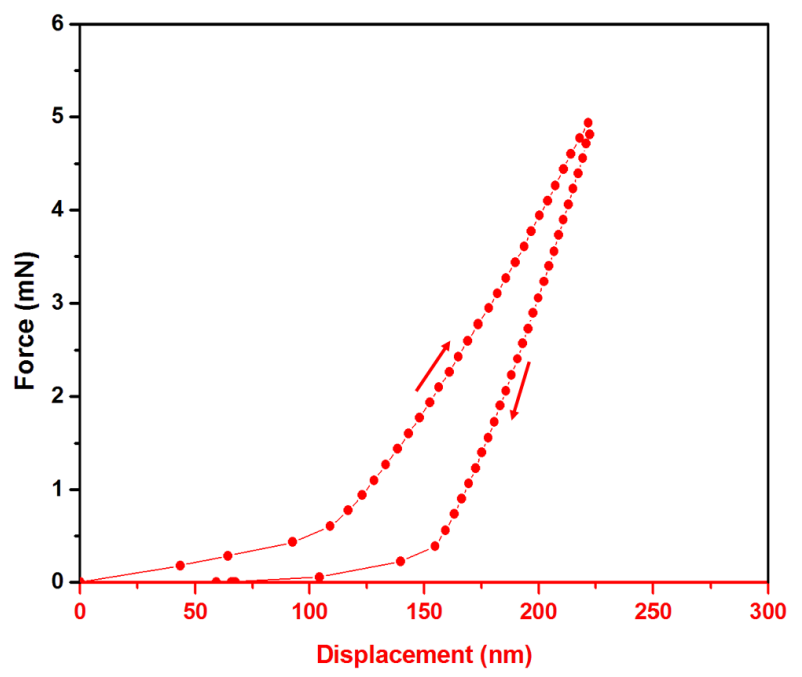

(b)

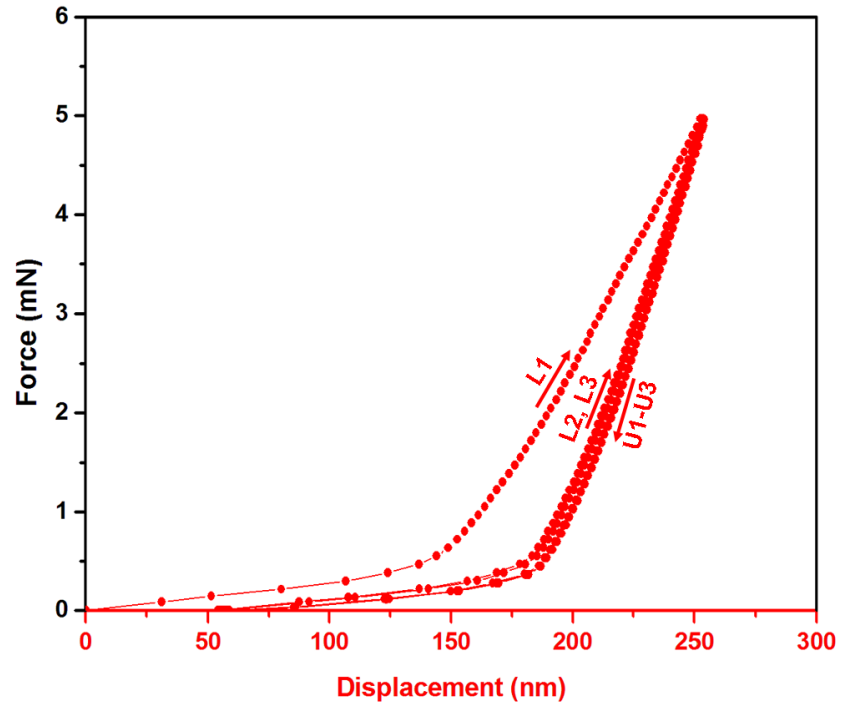

(c)

Figure 4. Representative load-unload curves for nanoindentation tests are presented for a single indent of the $700 \mathrm{~nm}$ thick film in (a) and single and three indents of the $1400 \mathrm{~nm}$ thick films in (b) and (c), respectively. The arrows indicate the load (L) and unload (U) cycles, with the three load-unload cycles indicated in (c). 


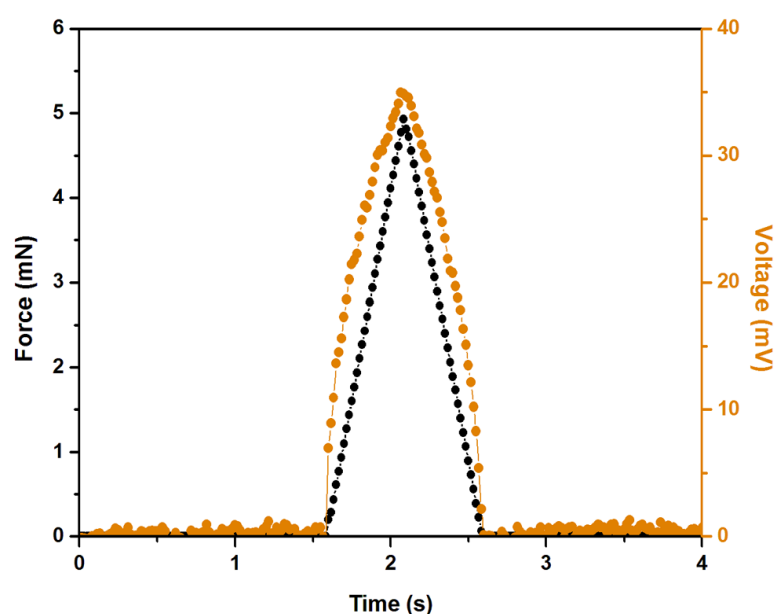

(a)

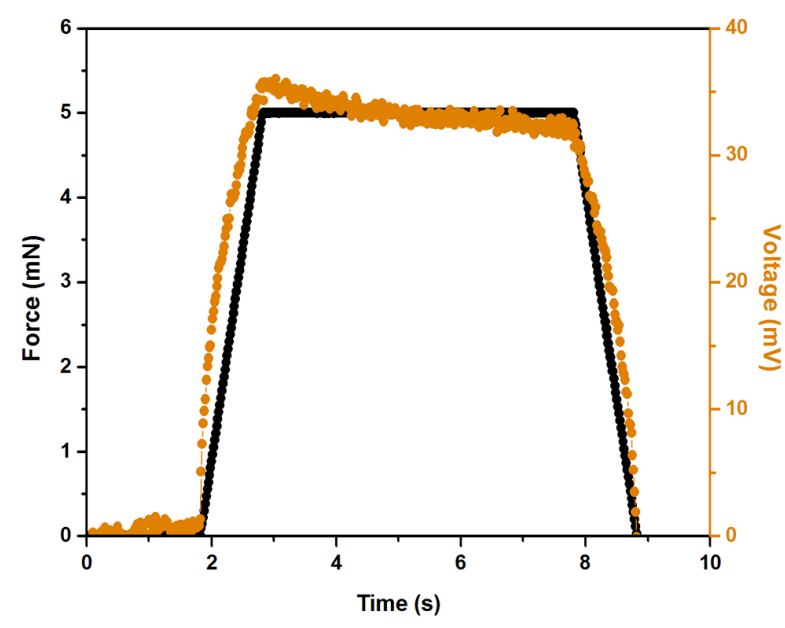

(b)

Figure 5. Piezoelectric voltage generation measured in situ under nanoindentation. Force (shown in black) in the form of a ramp function was applied on the thin film surface, and the generated voltage (shown in orange) at that point was measured using a conductive tip. The ability to perform accurate quantitative measurements is shown in (a) with a force of $5 \mathrm{mN}$ producing a voltage of $34.95 \mathrm{mV}$. Holding force at peak value after ramp up results in a sustained and slowly decaying voltage during that period, followed by a decrease with ramp down of force, as shown in (b).

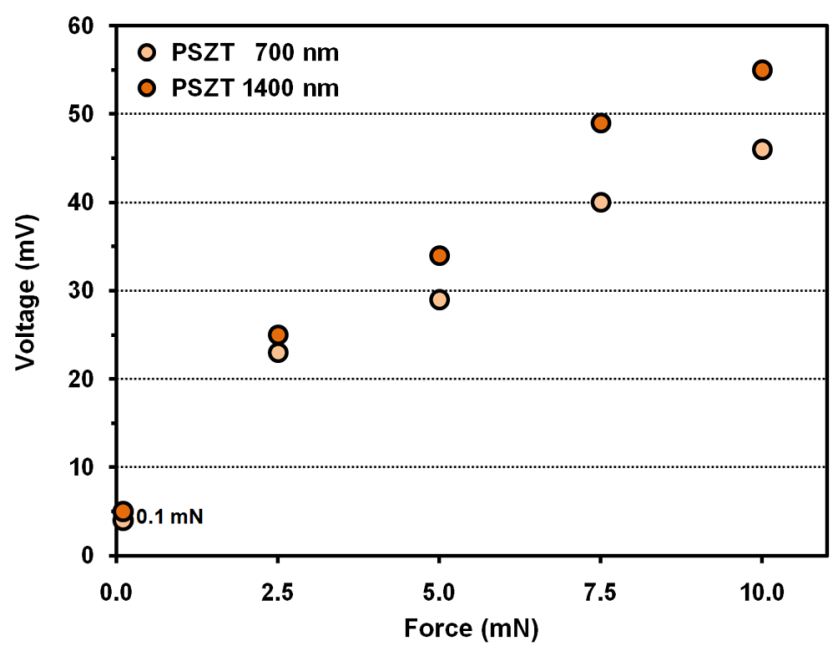

Figure 6. Comparison of voltage generation from the PSZT thin film samples of thickness $700 \mathrm{~nm}$ and $1400 \mathrm{~nm}$. The thicker sample produced $20 \%$ higher voltage for most measurements. 


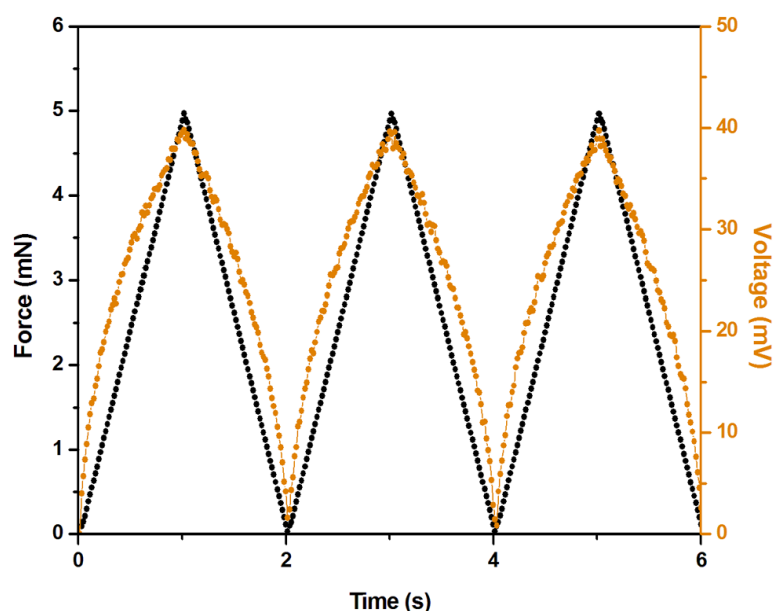

(a)

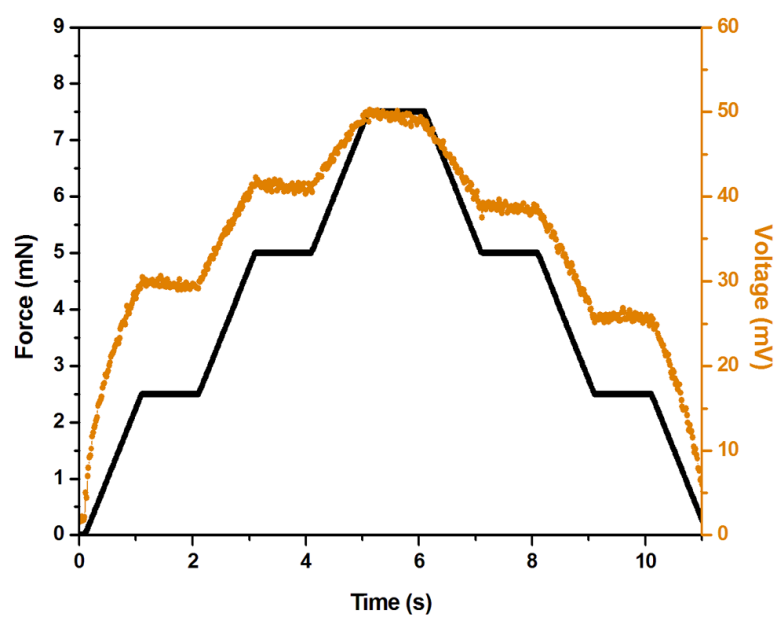

(c)
Submitted to

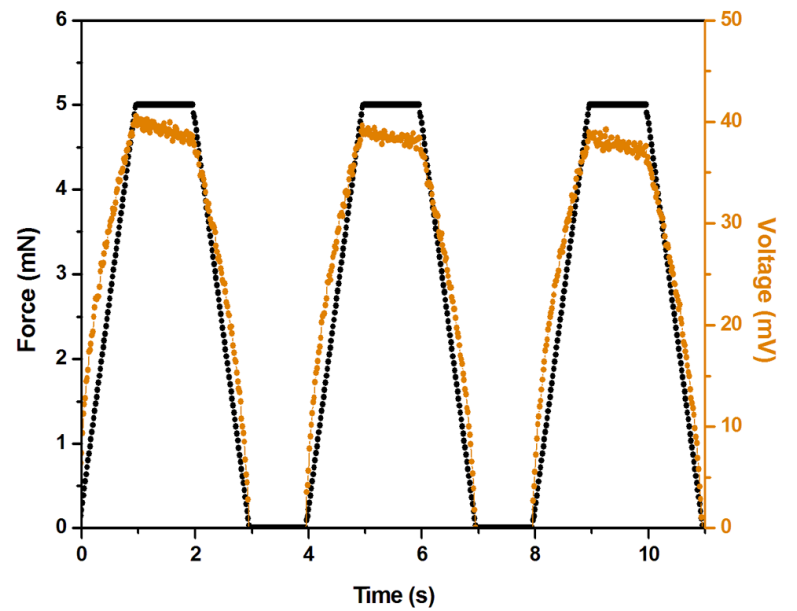

(b)

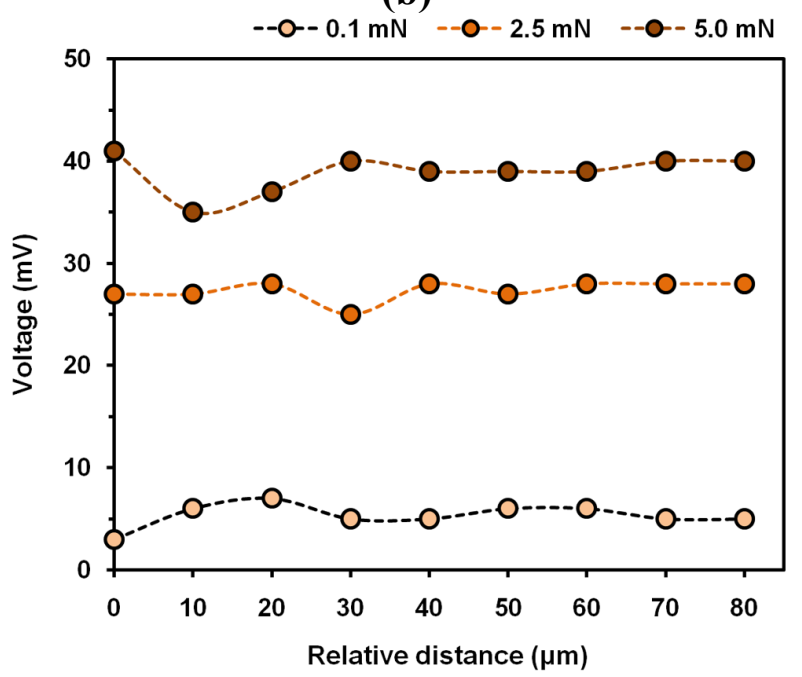

(d)

Figure 7. Piezoelectric voltage generation from nanoscale indentation under different force functions are shown in (a)-(c). These results highlight the repeatable voltage generation for multiple force cycles and the almost linear force dependence in the increase and decrease of generated voltage. Line mapping at $10 \mu \mathrm{m}$ intervals to study the variability in voltage generation for three different forces is shown in (d). 


\section{Submitted to \\ FUNCANCED \\ MATERIALS}

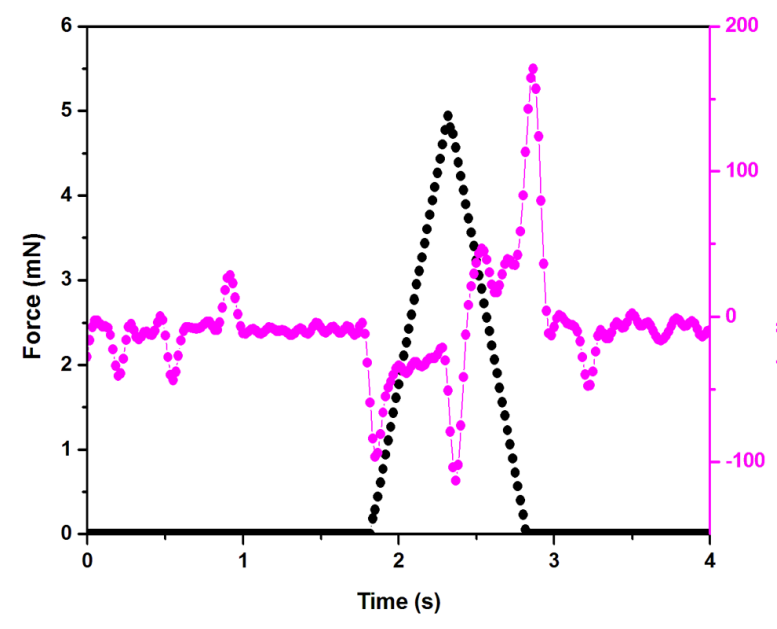

(a)

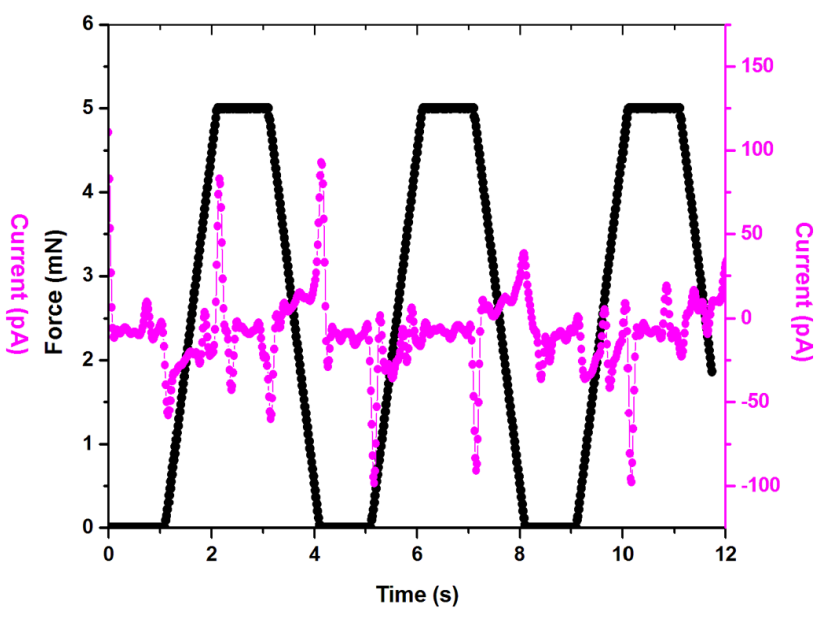

(b)

Figure 8. Current transients produced during nanoindentation of the $700 \mathrm{~nm}$ thick PSZT films are shown in (a). This short circuit measurement compares to the open circuit measurement shown in Figure 5(a). Transients at the three points of force variations can be observed. Compressive forces result in negative peaks and the return of the film to equilibrium produces a positive current. This positive transient has the maximum magnitude with a value of $173 \mathrm{pA}$. The repeatability of producing current transients can be observed in (b), with $\sim 100 \mathrm{pA}$ generated at most instances of force switching. 


\section{Submitted to}

Table 1. Comparison of voltage generation from thin films and nanoislands for forces of 0.1 and $2.5 \mathrm{mN}$. The results show that planar geometry has little influence on voltage generation.

\begin{tabular}{lcr}
\hline \multirow{2}{*}{ Sample Type } & \multicolumn{2}{c}{ Voltage (mV) } \\
\cline { 2 - 3 } & $\mathbf{0 . 1} \mathbf{~ m N}$ & $\mathbf{2 . 5} \mathbf{~ m N}$ \\
\hline $1400 \mathrm{~nm}$ thick films & 5 & 26 \\
$700 \mathrm{~nm}$ thick films & 4 & 22 \\
$400 \mathrm{~nm}$ diameter islands & 4 & 21 \\
$200 \mathrm{~nm}$ diameter islands & 4 & 22 \\
\hline
\end{tabular}

Table 2. Comparison of maximum transient current generated from the piezoelectric thin films and nanoislands for forces of 0.1 and $2.5 \mathrm{mN}$.

\begin{tabular}{lrr}
\hline \multirow{2}{*}{ Sample Type } & \multicolumn{2}{c}{ Current (pA) } \\
\cline { 2 - 3 } & $\mathbf{0 . 1} \mathbf{~} \mathbf{~ N}$ & $\mathbf{2 . 5} \mathbf{~} \mathbf{N}$ \\
\hline Thin films & 33.1 & 49.0 \\
$\mathbf{4 0 0} \mathrm{nm}$ diameter islands & 34.1 & 97.9 \\
$200 \mathrm{~nm}$ diameter islands & 95.5 & - \\
\hline
\end{tabular}

Table 3. Influence of different force loading rates on piezoelectric energy generation. Results presented are for the $700 \mathrm{~nm}$ thick PSZT film and a single indent to maximum force of $5 \mathrm{mN}$.

\begin{tabular}{rrr}
$\begin{array}{c}\text { Loading Rate } \\
\left(\mathrm{mN} \mathrm{s}^{-1}\right)\end{array}$ & \multicolumn{1}{c}{$\begin{array}{c}\text { Voltage } \\
(\mathrm{mV})\end{array}$} & \multicolumn{1}{c}{$\begin{array}{c}\text { Current } \\
(\mathrm{pA})\end{array}$} \\
\hline 1 & 32.55 & 20 \\
5 & 33.79 & 50 \\
10 & 34.95 & 170 \\
\hline
\end{tabular}




\section{Submitted to \\ FUNVANCED \\ MATERIALS}

\section{The table of contents entry}

We report on the use of nanoindentation to characterize in situ the voltage and current generation of piezoelectric thin films and nanoislands. This work presents the controlled observation of nanoscale piezoelectric voltage and current generation. The influence of size on energy generation parameters is reported demonstrating that nanoislands (see figure) can exhibit more effective current generation than continuous films.

Keyword: Stimuli-Responsive Materials

M. Bhaskaran,* S. Sriram, S. Ruffell, A. Mitchell

\section{Nanoscale Characterization of Energy Generation from Piezoelectric Thin Films}
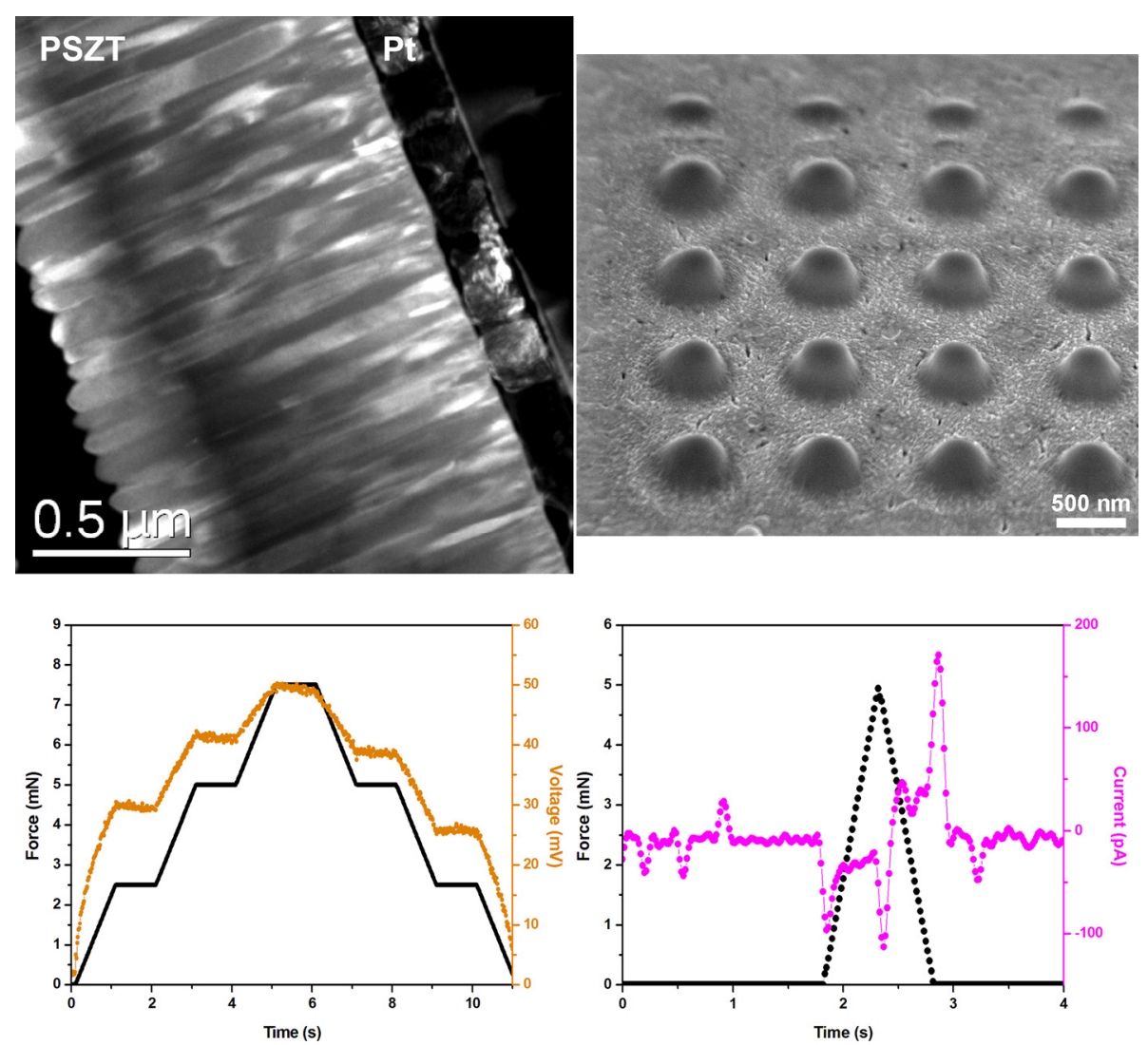